\section{Ambulante Rehabilitation Sucht: Rahmenbedingungen, Daten zur Entwicklung und Versicherten- einstellungen}

Joachim Köhler

Facharzt für Psychiatrie und Psychotherapie, Magister Public

Health, Fachbereich Medizin, BfA Berlin
Zusammenfassung: Die rechtlichen Rahmenbedingungen der ambulanten Rehabilitation Sucht und ihre Entwicklung in den letzten Jahren werden skizziert. Anhand von Daten zum Umfang der ambulant durchgeführten Entwöhnungsbehandlungen, zu wichtigen soziodemographischen Merkmalen und sozialmedizinischen Daten werden ambulante und stationäre Rehabilitation miteinander verglichen. Schließlich werden die Ergebnisse der Versichertenbefragung der BfA von 1997/1998 hinsichtlich der Erwartungen und Einstellungen zur ambulanten Rehabilitation dargelegt und kritisch diskutiert.

Schlüsselwörter: Ambulante Rehabilitation - Sucht Versichertenbefragung

Out-patient Rehabilitation Addiction: Legal Background, Data about Development and Attitudes of Patients: The legal background of out-patient addiction rehabilitation and its development in the last years are described. Out-patient and in-patient rehabilitation are compared on the basis of data about numbers of out-patient addiction rehabilitation, sociodemographic characteristics and social medical data. Finally outcomes of a survey of members of the german employees pension insurance 1997/1998 concerning anticipation and outlooks on out-patient rehabilitation are demonstrated and critically discussed.

Key words: Out-Patient Rehabilitation - Addiction - Survey

\section{Rechtliche Rahmenbedingungen}

Die stationäre Suchtrehabilitation findet ihre rechtliche Grundlage und nähere Ausgestaltung in der Empfehlungsvereinbarung von 1978, abgeschlossen von den Trägern der Kranken- und der Rentenversicherung.

Die stationäre Suchtrehabilitation wurde bereits lange Zeit vor der Empfehlungsvereinbarung durchgeführt. Die Empfehlungsvereinbarung regelte aber erstmalig dezidiert die Voraussetzungen, Inhalte sowie Anforderungen an die Ausstattung von stationären Suchtrehabilitationseinrichtungen. Sie

Suchttherapie 2000; 1: 137-142

(c) Georg Thieme Verlag Stuttgart · New York

ISSN 1439-9903 ist damit ein erfolgreiches Beispiel der Bestimmung von Schnittstellen und trägerübergreifender Kooperation.

Für die ambulante Rehabilitation fand die erste konkrete Umsetzung mit der Nachsorgeempfehlung vom 18.3.87 statt; diese Leistung kann zur Sicherung des Rehabilitationserfolges im Anschluss an eine stationäre Reha-Maßnahme erbracht werden.

Mit der Empfehlungsvereinbarung über Leistungen zur ambulanten Rehabilitation Sucht (EVARS) von 1991 wurde das Ziel verfolgt, die Durchführung ambulanter Leistungen zur Rehabilitation für Alkohol-, Medikamenten- und Drogenabhängige in qualifizierten Einrichtungen zu ermöglichen bzw. zu verbessern.

Das Besondere an dieser Vereinbarung war die erstmalige Einbeziehung der Drogenabhängigen in die ambulante Behandlungsform. Des Weiteren regelte die Vereinbarung dezidiert die Zugangsvoraussetzungen, die Leistungsinhalte, die Leistungsdauer und die pauschale Finanzierung der ambulanten Rehabilitation.

Die aktuell gültigen Regelungen zur ambulanten Rehabilitation der Kranken- und Rentenversicherungsträger liegen in der überarbeiteten Fassung der Empfehlungsvereinbarung der ambulanten Rehabilitation Suchterkrankter von 1996 vor.

In der neuen Fassung fanden im Wesentlichen folgende Ergänzungen Eingang:

- der so genannte Therapieverbund wurde geregelt,

- die Aufgabenbeschreibung des Arztes wurde konkretisiert.

Mit den Abgrenzungskriterien zwischen ambulanter und stationärer Rehabilitation, die von der Kranken- und der Rentenversicherung 1995 erstellt wurden, sind die Voraussetzungen und Anforderungsprofile an die ambulante Rehabilitation Suchterkrankter beschrieben.

In dem gemeinsamen Gremium der Kranken- und Rentenversicherung, der Arbeitsgemeinschaft Sucht, werden gegenwärtig die Empfehlungsvereinbarung und die damit verbundenen Anhänge diskutiert und aktualisiert. Als wesentliche Neuerung ist eine methadongestützte Rehabilitation für Drogenabhängige vorgesehen.

Die formalen Zugangskriterien für den Versicherten in eine ambulante Reha-Einrichtung entsprechen denen der stationären Rehabilitationsform: Neben den versicherungsrechtlichen 
Tab. 1 Durchgeführte Entwöhnungsbehandlungen. Quelle: Rehastatistik Datenbasis (1995-1997), abgerechnete Maßnahmen (1998 + 1999)

\begin{tabular}{|c|c|c|c|c|c|c|}
\hline & $\begin{array}{l}\text { Alte Bundesländer } \\
\text { ambulant }^{1}\end{array}$ & $\begin{array}{l}\text { Alte Bundesländer } \\
\text { stationär }\end{array}$ & $\begin{array}{l}\text { Neue Bundesländer } \\
\text { ambulant }^{1}\end{array}$ & $\begin{array}{l}\text { Neue Bundesländer } \\
\text { stationär }\end{array}$ & $\begin{array}{l}\text { gesamt } \\
\text { ambulant }^{1}\end{array}$ & $\begin{array}{l}\text { gesamt } \\
\text { stationär }\end{array}$ \\
\hline 1995 & 357 & 7187 & 27 & 698 & 385 & 7925 \\
\hline 1996 & 516 & 7411 & 28 & 758 & 548 & 8208 \\
\hline 1997 & 756 & 8023 & 47 & 980 & 816 & 9128 \\
\hline 1998 & 1210 & 9891 & 70 & 1286 & 1280 & 11177 \\
\hline 1999 & 1500 & 7768 & 110 & 1001 & 1610 & 8769 \\
\hline
\end{tabular}

1 ohne Nachsorgeleistungen

Voraussetzungen (also mindestens sechs anrechenbare Beitragsmonate) muss ein Sozialbericht vor Bewilligung der Maßnahme vorliegen. Darüber hinaus müssen Rehabilitationsbedürftigkeit und -fähigkeit sozialmedizinisch überprüft worden sein.

Die Zulassung der ambulanten Einrichtungen wird aktuell nicht nach Bedarfskriterien geprüft, sondern allein unter dem Aspekt, ob die Voraussetzungen, die nach der EVARS für das medizinisch-therapeutische Personal sowie das medizinischtherapeutische Konzept gefordert werden, erfüllt sind.

Die BfA nimmt bundesweit ca. 270 Beratungsstellen in Anspruch, die im Sinne der EVARS geprüft und zugelassen wurden.

\section{Daten zur Entwicklung der ambulanten Rehabilitation Sucht}

Die Auswertung der zu Lasten der BfA durchgeführten Maßnahmen zeigt, dass die Anzahl der ambulant durchgeführten Entwöhnungsbehandlungen in den letzten Jahren kontinuierlich gestiegen ist (Tab. 1).

Nach ursprünglich 5\% der insgesamt durchgeführten Entwöhnungsbehandlungen (1995) macht der Anteil der ambulanten Maßnahmen inzwischen über 15\% (1999) aus. Dabei ist die Zahl in den neuen Bundesländern 1999 mit ca. 10\% deutlich geringer als in den alten Bundesländern (Tab. 2).

Im Vergleich der soziodemographischen und rehabezogenen Merkmale zwischen ambulanter und stationärer Rehabilitation ergeben sich keine wesentlichen Unterschiede zwischen den beiden Gruppen. In der ambulanten Rehabilitation ist lediglich ein höherer Anteil von Verheirateten und ein geringerer Anteil von Geschiedenen oder Verwitweten zu

Tab. 2 Prozentsatz der ambulanten Behandlung an den durchgeführten Entwöhnungsbehandlungen. Quelle: RSD (1995-1997), abgerechnete Maßnahmen (1998 + 1999)

\begin{tabular}{lclc}
\hline & $\begin{array}{c}\text { Anteil amb. } \\
\text { alte Länder }\end{array}$ & $\begin{array}{l}\text { Anteil amb. } \\
\text { neue Länder }\end{array}$ & $\begin{array}{l}\text { Anteil amb. } \\
\text { gesamt }\end{array}$ \\
\hline 1995 & $4,7 \%$ & $3,7 \%$ & $4,6 \%$ \\
1996 & $6,5 \%$ & $3,6 \%$ & $6,3 \%$ \\
1997 & $8,6 \%$ & $4,6 \%$ & $8,2 \%$ \\
1998 & $10,9 \%$ & $5,2 \%$ & $10,3 \%$ \\
1999 & $16,2 \%$ & $9,9 \%$ & $15,5 \%$ \\
\hline
\end{tabular}

sehen. Bei der Entlassungsform und bei dem Anteil vorher durchgeführter Suchtrehamaßnahmen zeigen sich keine auffälligen Abweichungen (Tab. 3).

Die Rehabilitanden der ambulanten Behandlungsform haben deutlich weniger Zeiten der Arbeitsunfähigkeit und der Arbeitslosigkeit und dementsprechend auch einen höheren Anteil an lückenlos Beschäftigten in den zwei Jahren vor der Rehabilitation (Tab. 4).

Die ambulante Rehabilitation Sucht wird im bundesweiten Vergleich unterschiedlich häufig durchgeführt. Dies ist unter anderem in der Existenz und der Anerkennung entsprechender Einrichtungen begründet. Eine Übersicht über die einzelnen Bundesländer zeigt, dass die Zahlen, bezogen auf die Einwohnerzahl, sehr unterschiedlich ausfallen (Tab. 5).

Tab.3 Soziodemographische und rehabezogene Merkmale bei Sucht-Rehabilitanden 1998. Quelle: RSD 1998

\begin{tabular}{|c|c|c|c|c|}
\hline Merkmal & $\begin{array}{l}\text { stationär } \\
\text { Anzahl }\end{array}$ & Prozent & $\begin{array}{l}\text { ambulant } \\
\text { Anzahl }\end{array}$ & Prozent \\
\hline \multicolumn{5}{|l|}{ Alter } \\
\hline-34 Jahre & 2003 & $23 \%$ & 220 & $20 \%$ \\
\hline 35-44 Jahre & 3206 & $37 \%$ & 418 & $37 \%$ \\
\hline 45+ Jahre & 3431 & $40 \%$ & 479 & $43 \%$ \\
\hline gesamt & 8640 & $100 \%$ & 1117 & $100 \%$ \\
\hline Durchschnittsalter & 41,5 & & 42,5 & \\
\hline \multicolumn{5}{|l|}{ Geschlecht } \\
\hline männlich & 4949 & $57 \%$ & 601 & $54 \%$ \\
\hline weiblich & 3691 & $43 \%$ & 516 & $46 \%$ \\
\hline gesamt & 8640 & $100 \%$ & 1117 & $100 \%$ \\
\hline \multicolumn{5}{|l|}{ Familienstand } \\
\hline ledig & 2393 & $32 \%$ & 243 & $27 \%$ \\
\hline verheiratet & 3230 & $44 \%$ & 463 & $51 \%$ \\
\hline geschieden/verwitwet & 1775 & $24 \%$ & 194 & $22 \%$ \\
\hline gesamt & 7398 & $100 \%$ & 900 & $100 \%$ \\
\hline \multicolumn{5}{|l|}{ Entlassungsform } \\
\hline $\begin{array}{l}\text { regulär } \\
\text { vorzeitia }\end{array}$ & $\begin{array}{l}5999 \\
1951\end{array}$ & $\begin{array}{l}75 \% \\
24 \%\end{array}$ & $\begin{array}{l}795 \\
260\end{array}$ & $\begin{array}{l}15 \% \\
25 \%\end{array}$ \\
\hline vorzeitig & $\begin{array}{r}1951 \\
96\end{array}$ & $\begin{array}{r}24 \% \\
1 \%\end{array}$ & $\begin{array}{r}260 \\
5\end{array}$ & $\begin{aligned} 25 \% \\
0 \%\end{aligned}$ \\
\hline $\begin{array}{l}\text { verlegt } \\
\text { gestorben }\end{array}$ & $\begin{array}{r}96 \\
2\end{array}$ & $0 \%$ & 1 & $0 \%$ \\
\hline $\begin{array}{l}\text { gestorben } \\
\text { gesamt }\end{array}$ & 8048 & $100 \%$ & 1061 & $100 \%$ \\
\hline \multicolumn{5}{|c|}{ Sucht-Reha in den 60 Monaten vor Reha } \\
\hline nein & 7.322 & $85 \%$ & 974 & $87 \%$ \\
\hline ja & 1.318 & $15 \%$ & 143 & $13 \%$ \\
\hline gesamt & 8.640 & $100 \%$ & 1117 & $100 \%$ \\
\hline
\end{tabular}


Tab. 4 Arbeitsunfähigkeit, Arbeitslosigkeit und Beschäftigung in den zwei Jahren vor Rehabilitation bei Sucht-Rehabilitanden 1998. Quelle: RSD 1998

\begin{tabular}{|c|c|c|c|c|}
\hline Merkmal & $\begin{array}{l}\text { stationär } \\
\text { Anzahl }\end{array}$ & Prozent & $\begin{array}{l}\text { ambulant } \\
\text { Anzahl }\end{array}$ & Prozent \\
\hline \multicolumn{5}{|c|}{ Arbeitsunfähigkeit in den letzten 24 Monaten } \\
\hline nein & 6316 & $73 \%$ & 948 & $85 \%$ \\
\hline ja & 2324 & $27 \%$ & 169 & $15 \%$ \\
\hline gesamt & 8640 & $100 \%$ & 1117 & $100 \%$ \\
\hline \multicolumn{5}{|c|}{ Arbeitslosigkeit in den letzten 24 Monaten } \\
\hline nein & 5813 & $67 \%$ & 828 & $74 \%$ \\
\hline ja & 2827 & $33 \%$ & 289 & $26 \%$ \\
\hline gesamt & 8640 & $100 \%$ & 1117 & $100 \%$ \\
\hline \multicolumn{5}{|c|}{ lückenlose Beschäftigung in den 24 Monaten vor Reha } \\
\hline nein & 7020 & $81 \%$ & 797 & $71 \%$ \\
\hline ja & 1620 & $19 \%$ & 320 & $29 \%$ \\
\hline gesamt & 8640 & $100 \%$ & 1117 & $100 \%$ \\
\hline
\end{tabular}

Zusammenfassend ist festzustellen, dass die absolute Anzahl der ambulant durchgeführten Entwöhnungsbehandlungen und auch ihr prozentualer Anteil an den insgesamt durchgeführten Entwöhnungsbehandlungen in den letzten Jahren stetig zugenommen hat. Bei der genaueren Analyse soziodemographischer Merkmale ergeben sich keine wesentlichen Unterschiede zwischen den verschiedenen Behandlungsformen. Die Integration in das Arbeitsleben ist bei der ambulanten Rehabilitation vor der Maßnahme größer. Diese Unterschiede dürften im Wesentlichen Ausdruck einer positiven Selektion sein. Im Vergleich der einzelnen Bundesländer zeigt sich, dass die ambulante Rehabilitation, bezogen auf die absoluten Bevölkerungszahlen, deutlich unterschiedlich häufig durchgeführt wird.

\section{Einstellungen der Versicherten zur ambulanten Rehabilitation}

Es gibt bisher erst wenige Erkenntnisse, wie die ambulante Rehabilitation von den Betroffenen selbst aufgenommen wird. Nachdem zunächst in den angelsächsischen Ländern die Patientenzufriedenheit als ein Qualitätsmerkmal angebotener Leistungen identifiziert wurde [1,2], rücken die Aspekte der Nutzerbewertung auch in Deutschland zunehmend ins Blickfeld der Forschung und des Versorgungsalltags [3]. Untersuchungen zu Patientenerwartungen und -einstellungen liegen z.B. auf dem Gebiet der Orthopädie [4,5] vor, befassen sich aber eher retrospektiv nach einer durchgeführten Rehabilitation mit einer Bewertung des therapeutischen Prozesses und Ergebnisses. Obwohl die Rehabilitation von Suchtkranken das Indikationsgebiet ist, in dem die ambulante Rehabilitation am weitesten ausgebaut ist, und gleichzeitig als der Vorzeigebereich gilt, bei dem die Zusammenarbeit mit Selbsthilfegruppen und Angehörigen schon seit vielen Jahren zur Selbstverständlichkeit eines umfassenden Behandlungsansatzes gehört, ist über die Einstellungen der Klienten, Patienten oder Versicherten nach wie vor noch wenig bekannt.

Bei einer Versichertenbefragung der BfA und der LVA Sachsen, die 1997/1998 durchgeführt wurde, wurde aus dem Bestand der BfA-aktiv Versicherten eine repräsentative Auswahl von
Tab.5 Bewilligte Entwöhnungsbehandlungen je 10000 aktiv Versicherte, BfA 1999. Quelle: Infosys

\begin{tabular}{lrrr}
\hline Bundesland & stationär & ambulant & Summe \\
\hline \multirow{2}{*}{ Baden-Württemberg } & 7,8 & 2,9 & 10,7 \\
Bayern & 7,4 & 2,1 & 9,5 \\
Berlin & 7,7 & 2,2 & 10,0 \\
Brandenburg & 4,9 & 1,0 & 5,9 \\
Bremen & 10,3 & 3,8 & 14,1 \\
Hamburg & 8,2 & 3,1 & 11,3 \\
Hessen & 7,3 & 2,5 & 9,8 \\
Mecklenburg-Vorp. & 5,1 & 1,3 & 6,3 \\
Niedersachsen & 7,1 & 4,2 & 11,2 \\
Nordrhein-Westfalen & 6,5 & 2,5 & 9,0 \\
Rheinland-Pfalz & 7,2 & 2,1 & 9,3 \\
Saarland & 11,5 & 5,9 & 17,4 \\
Sachsen & 3,3 & 1,3 & 4,6 \\
Sachsen-Anhalt & 3,1 & 0,1 & 3,3 \\
Schleswig-Holstein & 7,0 & 3,5 & 10,5 \\
Thüringen & 4,5 & 0,6 & 5,1 \\
Summe & 6,7 & 2,4 & 9,1 \\
& & &
\end{tabular}

Personen zwischen 45 und 64 Jahren getroffen. Im September 1997 wurden 21898 Fragebogen versandt. Der Rücklauf betrug $48 \%[6,7]$.

Von den 7929 erfassten Versicherten ${ }^{1}$ beantworteten 178 $(2,2 \%)$ die Frage „Hatten oder haben Sie eine der folgenden Krankheiten?" positiv bei Sucht- oder Abhängigkeitserkrankungen. Diese Personengruppe wurde einer gezielten Auswertung unterworfen (im Folgenden als Suchtgruppe bezeichnet) und mit der „Restgruppe“ verglichen. Einschränkend muss hier darauf hingewiesen werden, dass in der Befragung nicht zwischen aktuellem und früherem Suchtmittelkonsum differenziert wurde. Es handelt sich hier also um zwei Personengruppen, die entweder noch ein akutes Suchtproblem haben und damit auch für eine ambulante Rehabilitation Sucht in Frage kämen oder die sich mit oder ohne Rehabilitation so weit stabilisiert haben, dass sie abstinent bleiben können. Auch wurde nur ein altersmäßig eingeschränkter Personenkreis befragt, Patienten von Suchtrehabilitation sind in der Regel jünger als 45 Jahre. Es handelt sich bei diesem Personenkreis im Wesentlichen um Alkoholkranke. Wegen dieser Einwände und der insgesamt nicht sehr großen Zahl von 178 Personen der Untergruppe dürfen die Ergebnisse aus wissenschaftlicher Sicht nicht überbewertet werden, sind jedoch für die derzeit geführte Diskussion über die Flexibilisierung der Rehabilitation wichtig und werfen einige Fragen für die Zukunft auf. Im Folgenden werden wichtige soziodemographische Merkmale und Einzelergebnisse verschiedener Fragen dargestellt:

Die Geschlechtsaufteilung zeigt bei der Suchtgruppe eine Betonung des männlichen Geschlechts (ca. 60\%), was bei der Prävalenz der Suchterkrankungen nicht anders zu erwarten war. In der Suchtgruppe ist eine etwas jüngere Altersverteilung als in der Restgruppe zu sehen. Auch beim Familienstand

\footnotetext{
${ }^{1}$ Nach Abzug der neutralen Ausfälle (unbekannt verzogen, verstorben o.Ä.) und von RentnerInnen
} 
zeigen sich Unterschiede, in der Suchtgruppe gibt es mehr Alleinstehende als in der Restgruppe. Die aktuelle Berufssituation war in beiden Gruppen vergleichbar, ca. 85\% waren jeweils berufstätig, ca. 15\% waren nicht berufstätig.

Bei der Suchtgruppe zeigt sich in der Selbsteinschätzung eine erhöhte Gefährdung der Erwerbsfähigkeit (22,6\% gegenüber 14,6\%; Chi²-Pearson: 0,01274). Bei den Erwerbstätigen war in der Suchtgruppe eine Häufung längerer Krankheitszeiten zu verzeichnen. Im Vergleich zur Restgruppe bezeichnete die Suchtgruppe ihren Gesundheitszustand im Allgemeinen eher als schlechter.

Bei der Frage, wie häufig alkoholische Getränke eingenommen werden, zeigen sich in der Suchtgruppe die beiden Untergruppen der Problemtrinker und der Abstinenten.

Schmerzmittel werden in den beiden Gruppen nicht signifikant unterschiedlich eingenommen. Schlaf- und Beruhigungsmittel werden in der Suchtgruppe jedoch häufiger konsumiert (z. B. 10,8\% gegenüber 3,6\% fast jeden Tag).

Der Hausarzt wird nicht unterschiedlich häufig konsultiert. Facharztbesuche sind in der Suchtgruppe etwas häufiger, statistisch jedoch nicht signifikant, zu konstatieren. Krankenhausaufenthalte sind in der Suchtgruppe häufiger. Die Befragten der Suchtgruppe haben tendenziell häufiger ambulante und stationäre Rehabilitationsmaßnahmen durchgeführt, die Abweichungen sind jedoch nicht statistisch signifikant. Erfahrungen mit Selbsthilfegruppen hatten beide Gruppen in recht unterschiedlichem Umfang, in der Suchtgruppe 37,1\%, in der Restgruppe lediglich 3,4\% der Befragten.

Die Mehrzahl der Befragten wünscht eine stationäre Rehabilitation, bei der Suchtgruppe sogar ein noch höherer Prozentsatz (Tab. 6).

Im Folgenden sind wichtige Aussagen und Einstellungen zur ambulanten und stationären Rehabilitation zusammengefasst Tab. 7).

Die Aussage, dass man sich einen Rehabilitationsort wünsche, der in der Nähe des Wohnortes liege, wird von beiden Untergruppen eher als falsch angesehen. Genauso wird die Aussage, dass man zur Rehabilitation in seiner gewohnten Umgebung bleiben möchte, mehrheitlich verneint, von der Suchtgruppe sogar noch häufiger; die Unterschiede zur Restgruppe sind jedoch nicht signifikant. Eine Mehrzahl der Befragten beider Gruppen findet zur Rehabilitation die Unterbringung in einer Klinik mit Vollverpflegung angenehm. Nur wenige Befragte messen der Möglichkeit, zu Hause zu übernachten, Gewicht bei; zwischen den Gruppen zeigen sich in

Tab. 6 Gewünschte Form der Rehabilitation

\begin{tabular}{|c|c|c|c|c|}
\hline & stationäre & ambulante & kombinierte & $\begin{array}{l}\text { weiß } \\
\text { nicht }\end{array}$ \\
\hline Suchtgruppe & $64,9 \%$ & $11,5 \%$ & $11,5 \%$ & $12,1 \%$ \\
\hline Restgruppe & $55,6 \%$ & $17,9 \%$ & $10,1 \%$ & $16,4 \%$ \\
\hline Signifikanz & Chi²-Pearson & 0,03085 & & \\
\hline
\end{tabular}

dieser Bewertung keine wesentlichen Unterschiede. Die Aussage, nicht von Familie und Freunden getrennt sein zu wollen, wird von beiden Gruppen eher abgelehnt.

In beiden Gruppen wird keine große Notwendigkeit gesehen, neben einer ambulanten Reha dringende private Dinge zu erledigen, in der Suchtgruppe sogar noch seltener. Auch beruflich dringend notwendige Angelegenheiten werden in beiden Gruppen gering gewichtet. Die Befürchtung, bei der ambulanten Reha sich nicht von den häuslichen Verpflichtungen befreien zu können, wird so mehrheitlich angegeben, in der Suchtgruppe sogar noch etwas häufiger; die Unterschiede sind jedoch nicht signifikant.

Die ärztliche/therapeutische Betreuung wird in der stationären Rehabilitation besser als in der ambulanten vermutet. Die Verknüpfung zu vorausgegangener oder nachfolgender Therapie scheint keine wesentliche Rolle zu spielen.

Die Mehrheit in beiden Gruppen ist der Meinung, einen neuen, der Gesundheit angepassten Lebensstil besser in der stationären Reha erlernen zu können; die Unterschiede zwischen beiden Gruppen sind nicht statistisch signifikant.

Zusammenfassend kann festgestellt werden, dass bei dieser repräsentativen Befragung von Versicherten allgemeine Einstellungen erfasst worden sind, die nicht unbedingt auf die typische Klientel von Suchtrehabilitationseinrichtungen übertragen werden können. So spiegelt die berufliche Integration von ca. $85 \%$ in beiden Untergruppen nicht den Alltag in den Behandlungsstätten wider.

Wesentliche Informationen der Auswertungen sind bekannt und überraschen nicht, so z.B. die Tatsachen, dass bei der Suchtgruppe

- es zu längeren Krankheitszeiten kommt,

- der Gesundheitszustand als schlechter eingeschätzt wird,

- eine erhöhte Gefährdung der Erwerbsfähigkeit besteht,

- vermehrt Schlaf- und Beruhigungsmittel eingenommen werden und,

- vermehrt Erfahrungen mit Selbsthilfegruppen bestehen.

Es stimmt jedoch nachdenklich, dass bei den Befragten der Wunsch nach einer stationären Rehabilitation gegenüber der ambulanten Rehabilitation so deutlich überwiegt. Wesentliche Vorteile der ambulanten Rehabilitation, wie z.B.

- die Nähe der Wohnung

- der Verbleib in der gewohnten Umgebung und die fehlende Trennung von Familie und Freunden,

- die Möglichkeit der Erledigung wichtiger privater oder beruflicher Dinge oder

- die Verknüpfung mit weiterer Therapie vor und nach der Rehabilitation,

werden nicht als wichtig angesehen. Vielmehr wird die Unterbringung in einer Klinik erwünscht, die ärztliche/therapeutische Betreuung wird im stationären Bereich als besser angesehen und die Befreiung von häuslichen Verpflichtungen wird als Entlastung empfunden. 
Tab. 7 Einstellungen zur ambulanten und stationären Rehabilitation

\begin{tabular}{|c|c|c|c|c|c|}
\hline & $\begin{array}{l}\text { Suchtgruppe } \\
\text { völlig oder } \\
\text { ziemlich richtig }\end{array}$ & $\begin{array}{l}\text { Restgruppe } \\
\text { völlig oder } \\
\text { ziemlich richtig }\end{array}$ & $\begin{array}{l}\text { Suchtgruppe } \\
\text { völlig oder } \\
\text { ziemlich falsch }\end{array}$ & $\begin{array}{l}\text { Restgruppe } \\
\text { völlig oder } \\
\text { ziemlich falsch }\end{array}$ & $\begin{array}{l}\text { Signifikanz } \\
\text { Chi²-Pearson }^{2}\end{array}$ \\
\hline Rehaort in der Nähe des Wohnortes & $19,9 \%$ & $22,7 \%$ & $55,7 \%$ & $49,2 \%$ & 0,51507 \\
\hline Reha in der gewohnten Umgebung & $13,7 \%$ & $19,3 \%$ & $65,7 \%$ & $55,2 \%$ & 0,09677 \\
\hline Unterbringung in einer Klinik angenehm & $76,1 \%$ & $70,9 \%$ & $8,0 \%$ & $8,8 \%$ & 0,11093 \\
\hline Möglichkeit, zu Hause zu übernachten & $18,2 \%$ & $22,1 \%$ & $62,5 \%$ & $54,1 \%$ & 0,29801 \\
\hline will nicht von Familie und Freunden getrennt sein & $14,8 \%$ & $19,2 \%$ & $63,7 \%$ & $53,6 \%$ & 0,12577 \\
\hline dringende private Dinge erledigen & $10,2 \%$ & $16,7 \%$ & $72,7 \%$ & $60,9 \%$ & 0,02646 \\
\hline dringende berufliche Dinge erledigen & $6,2 \%$ & $9,7 \%$ & $76,6 \%$ & $72,1 \%$ & 0,08754 \\
\hline $\begin{array}{l}\text { ambulant keine Befreiung von häuslichen } \\
\text { Verpflichtungen }\end{array}$ & $64,2 \%$ & $55,6 \%$ & $21,6 \%$ & $25,1 \%$ & 0,07396 \\
\hline $\begin{array}{l}\text { ärztliche/therapeutische Betreuung } \\
\text { stationär besser }\end{array}$ & $72,8 \%$ & $66,0 \%$ & $10,8 \%$ & $12,7 \%$ & 0,47203 \\
\hline $\begin{array}{l}\text { Verknüpfung zu vorausgegangener oder } \\
\text { folgender Therapie }\end{array}$ & $24,5 \%$ & $23,0 \%$ & $43,2 \%$ & $41,3 \%$ & 0,71867 \\
\hline neuer Lebensstil in der stat. Reha & $72,7 \%$ & $63,3 \%$ & $8,5 \%$ & $11,4 \%$ & 0,09411 \\
\hline
\end{tabular}

\section{Zusammenfassung und Ausblick}

Die ambulante Rehabilitation Sucht hat sich in den letzten Jahren sowohl bei den Anträgen, den bewilligten Maßnahmen wie auch bei den durchgeführten Maßnahmen kontinuierlich erhöht und nahm bei der BfA im Jahre 1999 bei den durchgeführten ambulanten Rehabilitationsmaßnahmen bundesweit einen Prozentsatz von etwas über $15 \%$ ein. Ziel der BfA ist es, diesen Anteil weiter auszubauen und jenen Versicherten eine ambulante Behandlung anzubieten, die diese Rehabilitationsform akzeptieren und in der Lage sind, in diesem Rahmen suchtmittelabstinent zu leben und die in der Empfehlungsvereinbarung beschriebenen Anforderungen zu erfüllen.

Dadurch können wesentliche Vorteile der ambulanten Rehabilitation genutzt werden, insbesondere die wohnortnahe therapeutische Arbeit unter Einbeziehung des Arbeitsplatzes und des sozialen Umfeldes. Weiterführende Maßnahmen können vorbereitet und nahtlos in die Behandlung integriert werden (ambulante ärztliche Versorgung, Besuch von Selbsthilfegruppen).

Als Fazit der Ergebnisse der Versichertenbefragung kann festgehalten werden, dass trotz einiger methodischer Bedenken, die gegen diese Auswertungen vorgebracht werden können, die ambulante Rehabilitation Sucht mit ihren Vorteilen und inhaltlichen Aspekten bei den Betroffenen leider noch nicht so angenommen wird, wie dies aus Sicht des Kostenträgers zu wünschen wäre. Stetig steigende absolute Zahlen von ambulanten Rehamaßnahmen und auch von ihrem prozentualen Anteil an den insgesamt durchgeführten Maßnahmen können nicht darüber hinwegtäuschen, dass es bei den Versicherten nach wie vor ein Akzeptanzproblem der ambulanten Rehabilitation gibt.
Der Bereich der Abhängigkeitserkrankungen spielt bei der ambulanten Rehabilitation die Vorreiterrolle bei der Rentenversicherung. In keinem anderen Indikationsbereich ist der Anteil der ambulant durchgeführten Maßnahmen so hoch. Nachdem in den letzten Jahren eine Vielzahl von Einrichtungen zur Durchführung der ambulanten Rehabilitation Sucht anerkannt worden ist, konzentrieren sich die gegenwärtigen Bemühungen auf eine einheitliche Dokumentation der durchgeführten Leistungen im Sinne einer Optimierung der Prozess- und Ergebnisqualität [8] sowie eine Beteiligung am Qualitätssicherungsprogramm der Rentenversicherung. Das im Bereich der stationären Suchtbehandlung inzwischen erfolgreich eingeführte Peer-Review-Verfahren muss neben den übrigen Instrumenten den spezifischen Bedingungen der ambulanten Rehabilitation angepasst werden.

Langfristig werden sich die z.Z. noch etwas starren Grenzen zwischen ambulanter, teilstationärer und stationärer Rehabilitation aufweichen müssen, indem den Betroffenen eine Rehabilitationsleistung im Sinne einer modularen Therapie angeboten wird, bei der Therapieangebote individuell angepasst und flexibel ausgerichtet sind. Dabei müssen Kliniken und ambulante Einrichtungen konzeptionell eng zusammenarbeiten und stärker als bisher vernetzt sein. Hierfür müssen entsprechende Therapieangebote entwickelt, valide differenzielle Indikationskriterien gefunden und eine selektive und adaptive Diagnostik durchgeführt werden. Auch die Rehabilitationsforschung muss hierfür ihren Beitrag leisten, um empirisch abgesicherte Entscheidungen zu ermöglichen. 


\section{Literatur}

${ }^{1}$ Hall J, Dornan MC. What patients like about their medical care and how often they are asked: A meta-analysis of the satisfaction literature. Soc Sci Med 1988; 27: 935-939

${ }^{2}$ Lebow JL. Research assessing consumer satisfaction with mental health treatment: A review of findings. Evaluation and Program Planning 1983; 6: 211-236

${ }^{3}$ Raspe H, Voigt S, Herlyn K, Feldmeier U, Meier-Rebentisch K. Patienten-,,Zufriedenheit“" in der medizinischen Rehabilitation. Gesundheitswesen 1996; 58: 372-378

${ }^{4}$ Gülich M, Jäckel WH. Die Patientensicht in der ambulanten orthopädisch/traumatologischen Rehabilitation. Die Rehabilitation 1999; 38 Supplement 1: S52-S60

${ }^{5}$ Riehemann W, Muthny FA, Freutel HJ, Ostermann HB, Paes P, Witting U. Einstellungen von orthopädischen Rehabilitanden zur stationären, teilstationären und ambulanten Rehabilitation. Prävention und Rehabilitation 1996; 8: 47-52

${ }^{6}$ Klosterhuis H, Stallmann M, Löffler HE, Wegscheider K. Subjektive Gesundheit, Rehabilitationsbedürftigkeit und inanspruchnahme im West-Ost-Vergleich - Ergebnisse einer Versichertenbefragung der BfA und der LVA Sachsen. Die Rehabilitation 1999; 38 Supplement 2: S128-S133

${ }^{7}$ Rische H, Löffler HE. Rehabilitationsbedarf höher als Rehabilitationsinanspruchnahme - Ergebnisse einer Versichertenbefragung der BfA und der LVA Sachsen. Die Angestelltenversicherung 1998; 45: 394-404

${ }^{8}$ Bundesversicherungsanstalt für Angestellte. Ambulante Rehabilitation Sucht - Anforderung, Entlassungsberichte, Zielsetzung -. Tagungsbericht der Fachtagungen am 11. und 18.3.2000 in Berlin

\section{Joachim Köhler}

Facharzt für Psychiatrie und Psychotherapie, Magister Public Health

BfA Berlin, Fachbereich Medizin

R 6207, Ruhrstraße 2

10407 Berlin

E-mail: joachim.koehler@bfa-berlin.de 